\title{
La Subdivisión de Medicina Familiar de la UNAM
}

\author{
Arnulfo Irigoyen-Coria, ' Lilia Aurora Arévalo-Ramírez ${ }^{* *}$
}

La Universidad Nacional Autónoma de México (UNAM) se fundó el 21 de septiembre de 1551 con el nombre de la Real y Pontificia Universidad de México, desde entonces ha desempeñado un papel protagónico en la historia y formación de México; actualmente, es considerada como la más grande e importante universidad de México e Iberoamérica. Tiene como propósito primordial estar al servicio del país y de la humanidad, y formar profesionistas útiles a la sociedad. Las tareas sustantivas de esta institución pública, autónoma y laica son la docencia, la investigación, principalmente acerca de las condiciones y problemas nacionales. Asimismo, extender con la mayor amplitud posible, los beneficios de la cultura ${ }^{1}$ y gracias a su labor, en el mundo académico es reconocida como una universidad de excelencia. La UNAM es un espacio de libertades, responde al presente y mira al futuro como el proyecto cultural más importante de México. En ella se practica cotidianamente el respeto, la tolerancia y el diálogo. La pluralidad de ideas y de pensamiento es apreciada como signo de su riqueza y nunca como factor de debilidad. ${ }^{2}$

\section{La Facultad de Medicina de la UNAM}

El origen de la Escuela Nacional de Medicina se remonta al siglo xvi con las bases de la Real y Pontificia Universidad de México en el año de 1553, aunque su antecedente inmediato fue en 1579 con la cátedra De prima, impartida por el doctor Juan de la Fuente. En 1960 la Escuela Nacional de Medicina se convirtió en facultad, institución que se caracteriza por su compromiso con la ciencia, el humanismo, la salud y el bienestar social. Por lo tanto, entre sus prioridades se encuentra la formación de médicos generales, especialistas, maestros y doctores altamente calificados, aptos para servir a la sociedad y ejercer el liderazgo científico, académico, asistencial y político de la medicina mexicana. ${ }^{3}$

\section{Acerca de la División de Estudios de Posgrado}

La División de Estudios de Posgrado (DEP) de la Facultad de Medicina ofrece el mayor número de programas de especialización en México, cuenta con 78 especializaciones médicas, de las cuales 21 son de ingreso directo a través del Examen Nacional de Aspirantes a Residencias Médicas (ENARM), las 57 restantes son de entrada indirecta y requieren de estudios previos de especialización. Algunos de estos programas son exclusivos de la UNAM y se imparten en las mejores instituciones de salud del país, pues cuenta con 92 sedes académicas donde se imparten 536 cursos de especialización.

Con la finalidad de asegurar la calidad de los programas de especialización, existe un Comité Académico (formado por especialistas de primer nivel), que actualiza los programas de especialización, vigila el cumplimiento del programa, mantiene comunicación abierta con profesores y alumnos para conocer sus opiniones e inquietudes y elabora los exámenes departamentales. Los programas son impartidos por 1414 profesores titulares y adjuntos, y cada unos de ellos está certificado por el consejo de su especialidad. De igual forma, la DEP otorga algunos servicios exclusivos como el acceso a laboratorios de investigación, centro de adiestramiento quirúrgico, empleo de modelos y simuladores en el Centro de Enseñanza por Simulación "Editor de la revista Atención Familiar; Subdivisión de Medicina Familiar, División de Estudios de Posgrado, Facultad de Medicina, Universidad Nacional Autónoma de México. ${ }^{* *}$ Coordinadora

Este artículo debe citarse: Irigoyen-Coria A, Arévalo-Ramírez LA. La Subdivisión de editorial de la revista Atención Medicina Familiar de la UNAM. Aten Fam. 2014;21(3):67-68.
Familiar 
de Posgrado (Cesip), laboratorio de diseño digital en tercera dimensión, así como eventos culturales y deportivos, seguro médico y acceso en línea a la biblioteca digital, en la que se pueden consultar 7300 revistas electrónicas, 13000 suscripciones a publicaciones científicas y técnicas, 162 bases de datos internacionales, 6000 libros electrónicos y 41000 tesis en texto completo. Cabe resaltar que las especializaciones médicas impartidas en la UNAM son la mejor opción para los egresados de todas las unidades tanto nacionales como extranjeras, lo que las ha convertido en la mejor opción para el estudio e investigación profesional de la Medicina. ${ }^{3}$

\section{Subdivisión de Medicina Familiar}

Es un organismo académico y de investigación de la División de Estudios de Posgrado de la Facultad de Medicina de la UNAm, que en coordinación con las instituciones del Sistema Nacional de Salud, tiene como objetivo promover el desarrollo de programas de formación de recursos humanos, capacitación, educación continua e investigación en medicina familiar, así como establecer un vínculo de comunicación entre los médicos familiares del país. ${ }^{4}$

\section{Misión}

La Subdivisión de Medicina Familiar, como instancia académica y de investigación, está dedicada a crear, preservar, desarrollar, interpretar y diseminar el cuerpo de conocimientos relacionados con la medicina familiar. Se orienta a formar médicos especialistas altamente calificados y competentes en su disciplina, para dar servicio a la sociedad y ejercer el liderazgo científico, académico, asistencial, administrativo y político de su especialidad. Desarrolla actividades docentes, de investigación, difusión y extensión, basadas en el conocimiento científico, calidad académica, capacidad de innovación, ética y humanismo. Prepara recursos humanos profesionales, especializados y competentes para el presente y el futuro, favoreciendo el aprendizaje autodirigido, la actualización permanente tanto de alumnos como de profesores, y el aprovechamiento de las nuevas tecnologías y estrategias educativas. Mantiene un compromiso permanente con la búsqueda de la preservación de la salud, la acción anticipada al daño, basada en un enfoque de riesgo, y la atención continua e integral de los problemas que afectan la salud de los individuos y sus familias en el contexto de la comunidad en la que se encuentran. ${ }^{4}$

\section{Visión}

Se concibe como un organismo de excelencia académica, con el más alto reconocimiento nacional e internacional; comprometido con la ciencia, el humanismo, la salud y el bienestar social, cuyos logros lo sitúan en el liderazgo académico de la medicina familiar mexicana; esto le ha permitido realizar una adecuada gestión del conocimiento, así como la generación de políticas y programas para el desarrollo de la medicina familiar en México. ${ }^{4}$

\section{Oferta Académica de la Subdivisión de Medicina Familiar}

La Subdivisión de Medicina Familiar otorga respaldo académico a profesores y alumnos del Curso de Especialización en Medicina Familiar y del Curso de Especialización en Medicina Familiar para Médicos Generales de las sedes académicas del Instituto Mexicano del Seguro Social (IMSs), Instituto de
Seguridad y Servicios Sociales de los Trabajadores del Estado (ISSSTE), Secretaría de Salud del Distrito Federal (SSDF) y del Instituto de Seguridad Social del Estado de México y Municipios (ISSEMYM). Este respaldo académico se otorga a través del subcomité académico de esta especialización, así como de las coordinaciones de docencia, investigación y evaluación del propio departamento. ${ }^{4}$

El personal académico del departamento participa en actividades docentes de los seminarios de atención médica, investigación y educación del Curso de especialización en Medicina Familiar del Plan único de especializaciones médicas; otorga asesoría-tutoría en investigación a profesores y alumnos; desarrolla, aplica y califica exámenes trimestrales y departamentales; supervisa el desarrollo de las actividades docentes y cumplimiento del programa académico del Curso de Especialización en Medicina Familiar. Participa en la impartición de cursos de educación continua para profesores y egresados del Curso de Especialización en Medicina Familiar. ${ }^{4}$

\section{Referencias}

1. Universidad Nacional Autónoma de México (UNAM). Acerca de la UNAm [Internet]. [Citado: 2014 May 7]. Disponible en: http://www.unam. $\mathrm{mx} /$ acercaunam/es/

2. Universidad Nacional Autónoma de México (UNAM). Qué es la UNAM [Internet]. [Citado: 2014 May 7]. Disponible en: http://www.unam.mx/acercaunam/ es/unam/index.html

3. Facultad de Medicina, UnAm. ¿Quiénes somos? [Internet]. [Citado: 2014 May 7]. Disponible en: http://www.facmed.unam.mx/marco/index. php?dir_ver $=23$

4. División de Estudios de Posgrado, Facultad de Medicina, UnAM. Subdivisión de Medicina Familiar [Internet]. [Citado: 2014 May 7]. Disponible en: http://www.fmposgrado.unam.mx/informacionGral/depMF.html 